\title{
Lie detection analysis on basis of fuzzy comprehensive evaluation
}

\author{
ZiLong Chen ${ }^{1}$, BoTao $\mathrm{Ma}^{2}$, ChengFeng Wu³, Lu Fan', and XinZheng Guo \\ ${ }^{1}$ Electrical Engineering College of Chongqing University, Chongqing, China \\ ${ }^{2}$ Electrical Engineering College of Chongqing University, Chongqing, China \\ ${ }^{3}$ Mechanical College of Chongqing University, Chongqing, China \\ ${ }^{4}$ Hong Shen College of Chongqing University, Chongqing, China \\ ${ }^{5}$ Hong Shen College of Chongqing University, Chongqing, China
}

Keywords: Fuzzy comprehensive evaluation ; Lie detection experiment ; Accuracy testing

\begin{abstract}
The article has presented the lie detection methods on basis of fuzzy comprehensive evaluation after analyzing disadvantages of traditional lie detection. This lie detection method has converted qualitative lie detection problems into quantitative calculation, while reducing the subjectivity of the experiment and improving the experimental precision for the effective solution of such problems as multiple parameters and difficult quantization during the lie detection experiment. Finally, the accuracy has been tested after using homemade polygraph testing. Since the accuracy of the fuzzy comprehensive evaluation is $90.6 \%$, the method has been proved to be effective.
\end{abstract}

\section{Background}

Lie detection technology has begun to play an increasingly important role in daily life and polygraph has been applied as an adjunct in the criminal investigation, interrogation and other industries. In addition, with the progress of sensing technology and the increasing maturity of lie detection theory, the lie detection technology is rapidly developing and has been promoted. Meanwhile, enterprises can also carry out lie detection to judge the employee honesty in daily life. As a kind of new technology, lie detection has been applied in more and more industries.

At present, lie detection technology is in need of improvement of experimental facilities and testing methods, and most lie detection results can't be treated precisely and effectively, for example: some polygraph just applies threshold value method to deal with the lie detection result, leading that experimental data cannot give full play to its value, which is not conducive to the progress of lie detection technology.

\section{Fuzzy comprehensive evaluation lie detection method}

The determination process for the lie detection result can be viewed as a multi-objective decision making, but the lie detection has no accurate functional relationship with lying, and the lie detection process is easy to be influenced by environmental factors, so the judgment for lie detection result has great ambiguity and empirical. To solve this problem, we propose a judgment for lie detection result based on fuzzy comprehensive evaluation method.

Multiple indicators have been regarded as the lie detection parameters for traditional lie detection technology. In addition, with the improvement of the accuracy of lie detection, lie detection parameters 
become more accurate. However, since lying has no accurate functional relationship with such lie detection parameters as breathing, heartbeat and others, it is predicted that these parameters have positive or negative correlation with lying. Therefore, it is unreasonable to apply a simple linear threshold value method to determine whether to lie or not in traditional lie detection experiment.

To solve this problem, the fuzzy comprehensive evaluation method is applied based on membership theory in the fuzzy mathematics to convert qualitative evaluation into the quantitative evaluation, thereby improving the rationality and accuracy of lie detection to try to avoid influence and interference from subjective factors. Ultimately, the behavior of lying can be predicted in a combination of the parameters of lie detection. In addition, in order to test the effectiveness of fuzzy comprehensive evaluation, self-made multi-parameter polygraph is used for testing, which has a good understanding of the accuracy of fuzzy comprehensive evaluation. Finally, it is found that the accuracy of the method is relatively higher, which indicates the usability of the fuzzy comprehensive evaluation method. The following is the specific steps of the fuzzy comprehensive evaluation method:

Determination of lie detection parameter set. In the actual lie detection process, in general, multiple lie detection parameters are selected as evaluation basis, and these parameters constitute a index set of the comprehensive evaluation system. And the set of factors is as following:

$$
U=\left\{u_{1}, u_{2}, \cdots, u_{n}\right\}
$$

Where ${ }^{u_{i}}$ indicates some measurement index such as breathing and heartbeat.

Determination of evaluation degree. Each parameter has a corresponding review, for example, when the variation amplitude of such parameters as respiration, body temperature, skin resistance and so on changes more than the threshold value, it can be considered lying, thereby judging the index as lying. Taking into account the actual situation can only be lying or not lying, so the evaluation result of each parameter is lying and not lying. Thus, the comment set for a different lie detection parameters as follows:

$$
V=\left\{v_{1}, v_{2}\right\}
$$

Where $v_{1}$ represents lying, and $v_{2}$ represents not lying.

Determination of the weight of different parameters. Functions of different parameters are quite different to the comprehensive evaluation, and there may be information redundancy among the different parameters, so it is necessary to analyze and judge the weight of different parameters reasonably for the determination of the most appropriate weight of each factor to get a reasonable comprehensive evaluation. The weight of different parameters can be written as A vector quantity:

$$
\mathbf{A}=\left[a_{1}, a_{2}, \cdots, a_{n}\right]
$$


Where $a_{i}$ indicates the weight of the ist parameter and matches $\sum_{i=1}^{n} a_{i}=1$. In order to facilitate solving the problem, the analytic hierarchy process can be used to construct their own judgment matrix, and then calculate the maximum eigenvalue, and determine the weights through the consistency testing finally.

Determination of fuzzy comprehensive judgment matrix. The judgment matrix of $u_{i}$ is written as:

$$
\mathbf{R}_{i}=\left[r_{i 1}, r_{i 2}, \cdots, r_{i m}\right]
$$

Vector quantity $\mathbf{R}_{i}$ is the fuzzy subset of membership $\mathbf{V}$. Similarly, the fuzzy comprehensive judgment matrix of different indexes is as following:

$$
\mathbf{R}=\left[\begin{array}{cccc}
r_{i 1} & r_{i 1} & \cdots & r_{i 1} \\
r_{i 1} & r_{i 1} & \cdots & r_{i 1} \\
\vdots & \vdots & \ddots & \vdots \\
r_{i 1} & r_{i 1} & \cdots & r_{i 1}
\end{array}\right]
$$

It can be seen that the matrix $\mathrm{R}$ is a fuzzy relation matrix mapping from $\mathrm{U}$ to $\mathrm{V}$. Elements in Matrix $\mathrm{R}$ indicate the degree of the importance between the different parameters, if the element $r_{i j}$ in the index $\mathrm{R}$ is greater than 0.5 , which illustrates index $\mathrm{i}$ is important than the index $\mathrm{j}$; if it is less than 0.5 , the index $\mathrm{j}$ is more important. Matrix can be used to determine which indicators are important.

Applying fuzzy evaluation for comprehensive judgment. If there is the fuzzy relationship $\mathbf{R}=\left(r_{i j}\right)_{n \times m}$, mapping from $\mathrm{U}$ to $\mathrm{V}$, the fuzzy conversion will exist:

$$
T_{R}: F(U) \rightarrow F(V)
$$

After conversion and the combination with the weight operator A and fuzzy judgment matrix R, the final results of fuzzy comprehensive evaluation vector can be got, denoted as:

$$
\mathbf{B}=\mathbf{A} \bullet \mathbf{R}
$$

Where $\mathrm{A}$ is the weight conversion operator for fuzzy conversion.

Finally, the paper analyzes the vector quantity B and then selects the comment of the highest numerical value as a comprehensive evaluation result, which can determine whether subjects is lying or not. Obviously, in actual lie detection test, the last comment is lying and not lying.

\section{Actual lie detection experiment}

Determination of the test parameters and weights. Self-made multi-channel physiological and psychological polygraph device can be used, the polygraph is used for monitoring heart rate, breathing, 
skin resistance change, therefore, the parameter set $\mathrm{U}=$ \{heartbeat, respiration, skin resistance $\}$ is selected to determine the weight of different parameters by the Analytic Hierarchy Process weight which is $\mathrm{A}=\{0.33,0.31,0.36\}$. It is known that if the weights of selected parameters are similar, which indicates there is less correlation between parameters and can reflect the physiological changes of subjects more effectively.

Determination of fuzzy comprehensive evaluation matrix. For each experimentally measured parameter, when it varies over $50 \%$, it can reflect subjects are lying, or the subjects are not lying. It should be noted that subjects may lie from the point of view on certain parameters, but in terms of the other parameters, the tested subjects don't lie, there are such reasons for this phenomenon as a lack of precision of experimental equipment and physical factors of the tested subjects (probably some physiological indicates reaction of some people are not sensitive enough). However, since the fuzzy comprehensive evaluation has considered the combined effect of several factors, it is possible to effectively solve the problem of inconsistent index change, and the final matrix of fuzzy comprehensive evaluation obtained as follows:

$$
\mathbf{R}=\left[\begin{array}{ll}
0.23 & 0.77 \\
0.41 & 0.59 \\
0.19 & 0.89
\end{array}\right]
$$

The matrix shows that parameters corresponding with heart rate and skin resistance parameters change significantly, which displays the tested subjects lie, however, since breathing change is not obvious, which cannot show the above result effectively. After analysis, the direct reason for the result may be that the tested subject has no obvious breathing change.

Conducting fuzzy comprehensive evaluation. The aforementioned weight vector A multiplies the comprehensive evaluation matrix $\mathrm{R}$ equals to the comprehensive judgment vector $\mathrm{B}$, which is shown as follows:

$$
\mathbf{B}=\mathbf{A} \bullet \mathbf{R}=\left[\begin{array}{ll}
0.2714 & 0.7574
\end{array}\right]
$$

From comprehensive evaluation vector, the probability of lying may be 0.7574 , and the probability of not lying is 0.2714 . Since the probability of lying is greater, which shows the tested subject is lying.

\section{Accuracy testing}

Select 140 volunteers to have standard lie detection testing for acquiring theoretical predictions and the real situation. And the theoretical predictions can be obtained by fuzzy comprehensive evaluation method and the real situation can be known by asking the tested subject. If the conclusion is the same, the lie detection testing is successful and the method is reliable and effective; on the contrary it shows that the method is unreasonable. After conducting standard tests for 140 people, the accuracy rate of lie detection is $90.6 \%$, indicating that the accuracy of lie detection after applying the fuzzy comprehensive evaluation is still very good and the method for the lie detection testing is effective and reasonable. This method can be used to improve accuracy and reduce errors caused by subjective factors in actual lie detection application. 


\section{References}

[1] Si shoukui,Sun Xiqing, Application of mathematical model, National Defence industry press, Beijing, 2001.8.

[2] Liang Xiaoru,Yin Chunwu, “An Method to determine the index of polygraph testspolygraph tests," Journal of Xi an University of Arts \& Science, vol. 13,No.1,2010 .

[3] Zhang Jijun,"Fuzzy Analytical Hierarchy Process," Fuzzy System and Mathematics, vol. 14,No.2,2000 .

[4] Shao-Kai Ni, "Analysis for the Seven Method of Determining Evaluation Index", South China Preventive Medicine, vol. 28, No.6,2002

[5]Ji-Guo Zhang, Model for Fuzzy Comprehensive Evaluation Based on Information Diffusion, Statistics and Decision Making, vol. 20,2007

[6] Jia-Hong He, Principle of Limited Application of Lie Detection Conclusion and Evidence, Jurisprudence of China, vol. 2,2002

[7] Gen-Yue Fu, Chang-Kai Chen, Research Status and Trend of Traditional Lie Detection Technology, Advances in Psychological Science, vol. 1,2003

[8] Hong-Li Zheng, Tong-Chun Ding, New Development of Lie Detection Technology, Journal of Chinese People's Public Security University (JCR Science edition), vol. 1,2007 .

[9] Zhang Bin, Discussion of the Development and Legal Attitude of American Lie Detection Technology Research, Oriental Law, ol. 6,2010. 